\title{
Deterministic models of breeding scheme designs that incorporate genomic selection
}

\author{
J. E. Pryce, ${ }^{* 1}$ M. E. Goddard, ${ }^{*} \dagger$ H. W. Raadsma, $\ddagger$ and B. J. Hayes ${ }^{*}$ \\ ${ }^{*}$ Biosciences Research Division, Department of Primary Industries Victoria, 1 Park Drive, Bundoora 3083, Australia \\ †Faculty of Land and Food Resources, University of Melbourne, Parkville 3010, Australia \\ †ReproGen-Animal Bioscience Group, Faculty of Veterinary Science, University of Sydney, PMB3, Camden 2570, Australia
}

\section{ABSTRACT}

A deterministic model to calculate rates of genetic gain and inbreeding was used to compare a range of breeding scheme designs under genomic selection (GS) for a population of 140,000 cows. For most schemes it was assumed that the reliability of genomic breeding values (GEBV) was 0.6 across 4 pathways of selection. In addition, the effect of varying reliability on the ranking of schemes was also investigated. The schemes considered included intense selection in male pathways and genotyping of 1,000 young bulls (GS-Y). This scheme was extended to include selection in females and to include a "worldwide" scheme similar to GS-Y, but 6 times as large and assuming genotypes were freely exchanged between 6 countries. An additional worldwide scheme was modeled where GEBV were available through international genetic evaluations without exchange of genotypes. Finally, a closed nucleus herd that used juvenile in vitro embryo transfer in heifers was modeled so that the generation interval in female pathways was reduced to 1 or 2 yr. When the breeding schemes were compared using a GEBV reliability of 0.6 , the rates of genetic gain were between 59 and $130 \%$ greater than the rate of genetic gain achieved in progeny testing. This was mainly through reducing the generation interval and increasing selection intensity. Genomic selection of females resulted in a 50\% higher rate of genetic gain compared with restricting GS to young bulls only. The annual rates of inbreeding were, in general, $60 \%$ lower than with progeny testing, because more sires of bulls and sires of cows were selected, thus increasing the effective population size. The exception was in nucleus breeding schemes that had very short generation intervals, resulting in higher rates of both gain and inbreeding. It is likely that breeding companies will move rapidly to alter their breeding schemes to make use of genomic selection because benefits to the

Received March 12, 2010.

Accepted July 19, 2010.

${ }^{1}$ Corresponding author: jennie.pryce@dpi.vic.gov.au breeding companies and to the industry are considerable.

Key words: genomic selection, breeding program, genetic gain, inbreeding

\section{INTRODUCTION}

The potential to make selection decisions on reliable genomic estimated breeding values (GEBV) early in the life of animals, rather than waiting for phenotypes to become available, has substantial implications for the design of dairy cattle breeding programs (Schaeffer, 2006).

Genomic estimated breeding values combine genomic and pedigree information (Hayes et al., 2009). The reliability of GEBV in several countries significantly exceeds the reliability of parent average or sire pathway values, but currently is not as high as the reliability achieved in progeny testing (e.g., Harris et al., 2008; Hayes et al., 2009; Moser et al., 2009; VanRaden et al., 2009). Consequently, some breeding companies are in the process of adapting their breeding schemes to incorporate genomic information while continuing to progeny test at the same time (e.g., Harris et al., 2008).

If GEBV are used to make early selection decisions instead of progeny testing, modeling suggests that genetic gains could be increased by 30 to $217 \%$ over current rates of genetic gain (Schaeffer, 2006; Harris et al., 2008; König et al., 2009), mainly through reducing generation intervals. Another strategy, recently discussed by König and Swalve (2009), is to use GEBV to reduce the number of progeny-test daughters required. Thus, a desired accuracy is achieved where the reduction in number of progeny required is dependent on the heritability of the trait and the reliability of the GEBV.

The low cost of genotyping relative to progeny testing and the fact that the genomic information can be acquired early in the life of selection candidates make a range of alternative breeding schemes attractive with genomic selection (GS). For example, young bulls could be selected as sires of cows using GEBV only, or the number of bulls selected as progeny-test candidates 
could be reduced. These approaches can be extended or redesigned.

One such scheme could take advantage of the fact that dairy cattle breeding is a global industry. Breeding objectives in different countries are currently closely aligned (Miglior et al., 2005) and considerable exchange of genetic material occurs, primarily semen from progeny-tested sires. However, in many cases, it will not be possible for a single country or organization to have a reference population with enough performance records of their sires' daughters in other countries to derive prediction equations for genomic breeding values across all the countries of interest.

If the same SNP panel is used for genomic predictions and free exchange of candidate genotypes occurs between countries, then the same reliability can be achieved for bulls from foreign countries as for domestic sires (within a country). Alternatively, genotypes and phenotypes may not be shared across countries. In this case, GEBV could be converted to domestic scales using an adaptation of traditional multi-trait across-country evaluation (MACE) to handle nonindependent data, such as genomic MACE (GMACE; VanRaden and Sullivan, 2010). If this were applied, the reliabilities of bulls from foreign countries would be lower than that of domestic sires. Furthermore, the existence of genotype by environment interactions $(\mathbf{G} \times \mathbf{E})$ between countries could mean that some bulls that would not be selected in their country of birth may rank highly in another country. However, it is likely that most animals selected will either come from the country itself or from those countries exhibiting little $\mathrm{G} \times \mathrm{E}$ with the importing country. This type of scheme may also be of interest to countries that have no breeding programs of their own or are importers of semen from countries with similar breeding objectives.

Other possible breeding schemes include nucleus breeding schemes where elite herds of cows are mated to top sires to breed potential future sires. This type of scheme would probably be owned and regulated by breeding companies and is attractive because selection intensities can be increased in both male and female pathways. Such scenarios can also make use of reproductive technologies such as sexed semen or multiple ovulation and embryo transfer (MOET). Reproductive technologies may be more effective in achieving high rates of genetic gain in well-organized breeding schemes, rather than in commercial herds, provided that the technology is strategically used. Abdel-Azim and Schnell (2007) explored breeding schemes using MOET and sexed semen in conjunction with marker-assisted selection (but not genomic selection). They found an $11 \%$ increase in response to selection. Therefore, the combination of such reproductive technologies and genomic selection could improve the rate of genetic gain.

The classic response to selection $(\boldsymbol{\Delta} \mathbf{G})$ equation (e.g., Falconer, 1989) modified to include 4 pathways of selection is

$$
\Delta G=\frac{\sum_{i=1}^{4} I_{i} r_{i} \sigma_{A}}{\sum_{i=1}^{4} L_{i}},
$$

where $L_{i}$ is the generation interval in the $i$ th selection pathway, $I_{i}$ is the selection intensity in the $i$ th pathway of selection, $r_{i}$ is the accuracy of selection, and $\sigma_{A}$ is the genetic standard deviation of selection.

Using this equation, it follows that response to selection is affected by

1) The generation interval $(\mathbf{L})$. This is the average age of parents at the birth of their selected progeny (Falconer, 1989). In conventional breeding schemes that use progeny testing to generate data to calculate EBV, the generation interval in male pathways $\left(\mathrm{L}_{\mathrm{s}}\right)$ is long (e.g., Schaeffer, 2006). By using GEBV, $\mathrm{L}_{\mathrm{s}}$ can be reduced substantially and is dependent on the sexual maturity of bull calves. There is also scope to reduce the generation interval in female pathways $\left(\mathrm{L}_{\mathrm{f}}\right)$. For example reproductive technologies such as juvenile in vitro fertilization and embryo transfer (JIVET) can be used to obtain oocytes before sexual maturity (Raadsma and Tammen, 2005), potentially leading to a large reduction in generation interval.

2) The accuracy of the GEBV. This is mainly dependent on the size of the reference population available (e.g., VanRaden et al., 2009) to derive the GEBV prediction equations. Using current technology, the reliability of GEBV of production traits reported for 4 populations (Australia, New Zealand, the Netherlands, and United States/ Canada) range between 0.16 and 0.67 (Harris et al., 2008; Hayes et al., 2009; VanRaden et al., 2009).

3) Selection intensity and the number of animals genotyped. There may be diminishing returns to genotyping more animals if population size is a limiting factor.

The aim of this study was to evaluate a range of alternate breeding program designs incorporating genomic selection and reproductive technologies for both rate 
of genetic gain and effect on inbreeding. The breeding schemes investigated included genotyping of large numbers of young bull calves within a single country or across several cooperating countries and nucleus breeding schemes that focus on selection of young females facilitated by reproductive technologies. The reliability of GEBV used for most comparisons was 0.6. The effects of varying the reliability of GEBV were also investigated (0.1 to 0.9 ) because the reliability of GEBV may vary with size of training populations and across different traits (Hayes et al., 2009; Moser et al., 2009).

\section{MATERIALS AND METHODS}

The Rendel and Robertson (1950) method of calculating annual genetic gain using 4 pathways of selection was used. The same approach was applied by Schaeffer (2006) and König and Swalve (2009) to calculate response to selection under genomic selection. The 4 pathways are sires of bulls (SB), sires of cows (SC), dams of bulls (DB), and dams of cows (DC). The method was extended in the present study to model alternative schemes. Deterministic modeling was used and a single trait considered. The single trait could be considered an aggregate breeding value derived from a selection index.

\section{Model}

The default reliability of GEBV used was 0.6 , following Meuwissen et al. (2001), who used simulated data to show how breeding values could be predicted with accuracies as high as 0.8 , which is a reliability of 0.64 . This also aligns with deterministic predictions based on a reference population of 7,000 progeny-tested sires (Goddard, 2009). The effect of varying the reliability from 0.1 to 0.9 on response to selection was also investigated. All results presented in this paper are in genetic standard deviation units.

\section{Breeding Schemes}

The dairy population described in the scenarios was designed to represent the current situation in Australia of around 1.7 million cows (http://www.dairyaustralia. com.au/Our-Dairy-Industry/Industry-Statistics.aspx).

To be eligible as a potential bull dam (breeding cow), a female had to be pedigree registered and enrolled in a milk recording scheme. According to M. Shaffer (Holstein Australia, Melbourne, Australia; personal communication), the population of Holsteins that qualify as breeding cows in Australia is approximately 140,000 (this is based on approximately 50,000 calves registered annually, a $25 \%$ replacement rate, and $70 \%$ participation in milk-recording).
For schemes that included female pathways of selection, it was assumed that truncation selection was across multiple normal distributions representing age classes. The percentages of females in each of 6 age classes were $25,21,18,15,13$, and $8 \%$ (this represents the distribution of females across age classes in Australia). In progeny testing, the youngest age a cow could become a parent of the next generation was $3 \mathrm{yr}$ of age. The younger age classes were genetically superior to older age classes and the difference between consecutive age classes was equivalent to the annual rate of genetic gain in the scenario being evaluated. The reliability of EBV in each age class was calculated as $\frac{n h^{2}}{1+(n-1) r e}$ (e.g., Cameron, 1997), where $n$ is the number of lactation records available and assuming the heritability $\left(h^{2}\right)$ was 0.3 and repeatability (re) was 0.5 . The resulting reliability will be an underestimate because it excludes the contribution of pedigree.

Truncation selection was calculated across age classes. The selection intensity in the dam to breed bull pathway $\left(I_{D B}\right)$ was calculated as $I_{D B}=\sum_{i=1}^{6} I_{i} \times c_{i}$, where $I_{i}$ is the selection intensity in the $i$ th age class and $c_{i}$ is the contribution of age class $i$ to the total number of selected individuals. Similarly, the generation interval in the DB pathway $\left(L_{D B}\right)$ was calculated as $L_{D B}=\sum_{i=1}^{6} L_{i} \times c_{i}$, where $L_{i}$ is the age when offspring are born in the $i$ th age class. The contribution of age class $i$ to the total number of selected individuals $\left(c_{i}\right)$ was calculated using the method of Ducrocq and Quaas (1988). In brief, the animal is selected if $x>T_{x}$, so

$$
\begin{aligned}
\operatorname{Pr}(\text { the animal is selected }) & =\operatorname{Pr}\left(x>T_{x}\right) \\
& =\Phi \times\left(\frac{T_{x}-\mu}{r}\right),
\end{aligned}
$$

where $T_{x}$ is the truncation selection point, $x$ is the EBV of the selection candidate, $\Phi$ is the cumulative normal distribution function, $\mu$ is the mean of the age group, and $r$ is the accuracy; note: $\mu$ and $r$ depend on the animal's age. The genetic variance was assumed to be 1 .

\section{Descriptions of the Breeding Schemes}

Verbal descriptions of the breeding schemes are shown in Table 1 . The parameters used in this study are described in Table 2 .

$\boldsymbol{P T}$. This scheme is conventional progeny testing that includes age classes for dams. Three hundred and 
Table 1. A descriptive overview of the breeding schemes compared in this study

\begin{tabular}{ll}
\hline Scheme & Description \\
\hline PT & $\begin{array}{l}\text { Progeny testing of } 100 \text { bulls; } 2 \text { bulls graduate as sires to breed bulls (SB) and } 10 \text { bulls graduate as sires to breed cows (SC). } \\
\text { GS-PT }\end{array}$ \\
Genomic selection applied to progeny testing. Fifty bulls were progeny tested from 1,000 genomically \\
GS-Y & $\begin{array}{l}\text { Gelected bulls; } 2 \text { bulls graduate from progeny testing as SB and } 10 \text { bulls graduate as SC. } \\
\text { GS-Y-DC } \\
\text { Genomic-selection of } 1,000 \text { bull calves; } 20 \text { bulls graduate as SC and SB and have progeny when they reach } 2 \text { yr of age. }\end{array}$ \\
GS-WW & $\begin{array}{l}\text { Additionally, selection intensity is applied to the dams of cows (DC) pathway through selection of heifers and cows. } \\
\text { "Worldwide" scheme of } 6 \text { participating countries each with equal domestic population size. The participating }\end{array}$ \\
& $\begin{array}{l}\text { countries genotype } 1,000 \text { bulls each and the domestic country uses the best } 20 \text { as SB and SC regardless of country } \\
\text { GS-CN1 origin. The reliability of genomic EBV was } 0.6 \text { in the domestic country and } 0.8 \times 0.6 \text { in other countries. }\end{array}$ \\
GS-CN2 & $\begin{array}{l}\text { A nucleus of } 300 \text { females participating in juvenile in vitro fertilization and embryo transfer (JIVET) and have } \\
\text { progeny when they reach } 1 \text { yr of age. Only } 1 \text { round of JIVET is possible because of practical limitations. }\end{array}$ \\
& $\begin{array}{l}\text { The same as GS-CN1 except heifers are } 2 \text { yr of age when they have progeny. There is more } \\
\text { opportunity for JIVET and therefore double the offspring of GS-CN1 are produced. }\end{array}$ \\
\hline
\end{tabular}

thirty-three dams were required to generate 100 male calves selected for progeny testing from a population of 140,000 breeding cows (assuming a male to female ratio of $50 \%$ and a calving rate of $60 \%$ ). A calving rate of $60 \%$ was chosen because this reflects the typical proportion of cows calving within the first $42 \mathrm{~d}$ of the calving season (Dairy Research and Development Corporation, 2000; Haile-Mariam et al., 2003). In typical split and seasonal calving systems in Australia, most cows are inseminated with AI bulls in the first 6 wk of the AI period and this is then followed by a period of natural mating.

In the DB and DC pathways, cows were at least $3 \mathrm{yr}$ of age when their offspring were born. In conventional $\mathrm{PT}$, more intense selection occurs in the SB pathway than in the SC pathway, because most bull calves that enter PT are the result of planned matings. Therefore, annually, the top 2 males were selected as SB and the top 10 males were selected from 100 progeny-tested sires as SC, assuming the reliability of selection was

Table 2. Selected individuals versus candidates for selection, reliability, and pathway-specific generation interval $\left(L_{i}\right)$ for the pathways of selection $(\mathrm{SB}, \mathrm{SC}, \mathrm{DB} \text {, and } \mathrm{DC})^{1}$ considered for various breeding scheme designs

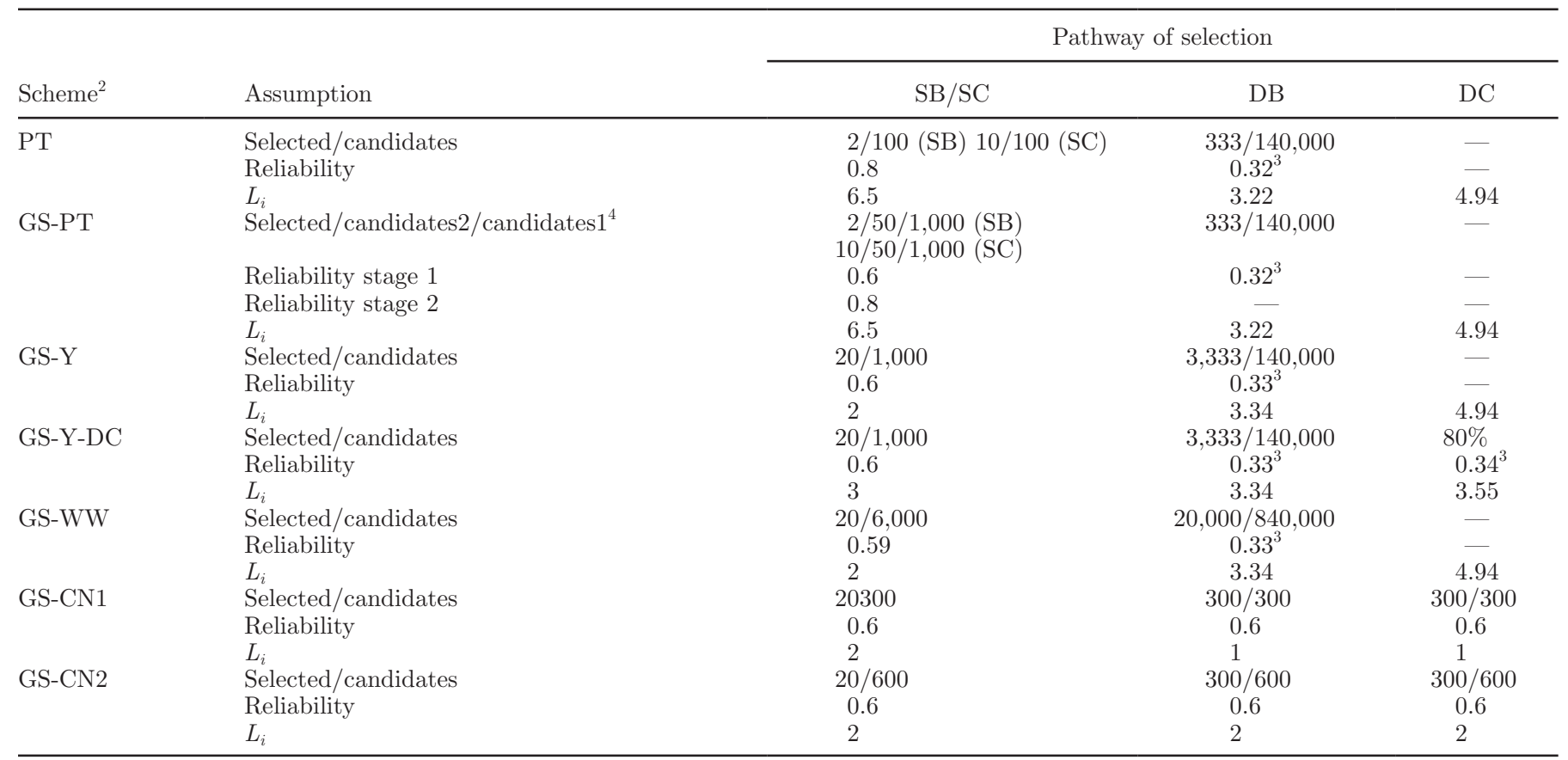

${ }^{1}$ Sires of bulls (SB), sires of cows (SC), dams of bulls (DB), and dams of cows (DC).

${ }^{2}$ See Table 1 for a description of each scheme.

${ }^{3}$ Reliability is the weighted mean across classes.

${ }^{4}$ Selected/candidates2/candidates1: selected individuals from candidates in stage 2 of selection and candidates at stage 1 of selection. 
0.8. An assumption of no selection in the DC pathway was made.

$\boldsymbol{G S} \boldsymbol{S} \boldsymbol{P T}$. This scheme was similar to PT, except that the number of sires that were progeny tested was 50 . For all GS schemes, the number of males that were genotyped was 1,000 . The top 50 out of 1,000 bulls were selected using GEBV. When the PT daughters of these sires have lactation records, the top 2 would be selected as SB and the top 10 for SC. The GS-PT scheme included 2 stages of selection in the sire pathways, the first was on GEBV and the second on EBV following progeny testing. After truncation selection following stage 1 of selection, the selected population is no longer normally distributed. This makes prediction of response to both stages of selection more complicated than with single-stage selection (Cunningham, 1975). Using the same approach as Jopson et al. (2004), the intensity of selection after 2 stages of selection was calculated using a standard bivariate normal distribution (SBN):

$$
S B N(x, y, \rho)=\frac{1}{2 \pi \sigma_{x} \sigma_{y} \sqrt{1-\rho^{2}}} \exp ^{\frac{-1}{2\left(1-\rho^{2}\right)}\left(\frac{x^{2}}{\sigma_{x}^{2}}+\frac{y^{2}}{\sigma_{y}^{2}}-2 \rho x y\right)},
$$

where $x$ and $y$ are EBV at stage 1 and 2 respectively, and $\rho$ is the correlation between the EBV at stage 1 and 2 . The variance at stage $2\left(\sigma_{y}^{2}\right)$ of selection was a function of the variance at stage $1\left(\sigma_{x}^{2}\right)$; that is, $\sigma_{y}^{2}=\left(1-\rho^{2}\right) \sigma_{x}^{2}$. The truncation point for the first stage of selection was calculated using the inverse of the standard normal cumulative distribution. Solving the truncation point $\left(T_{y}\right)$ in the second stage of selection required numerical integration. The selection intensity $(I)$ after the second stage of selection was

$$
I=\frac{\int_{T_{x}}^{\infty} \int_{T_{y}}^{\infty} S B N(x, y, \rho) \cdot x \mathrm{~d} y \mathrm{~d} x}{p_{y}},
$$

where $T_{x}$ is the truncation point for the first stage of selection and $p_{y}$ is the proportion selected after the second stage.

The schemes described in the next 2 sections include intense selection in either the male or female pathways. In all GS schemes, it was assumed that there were $20 \mathrm{SB}$ and $20 \mathrm{SC}$. This was because the reliability of GEBV in GS schemes (0.6) was lower than the reliability of EBV available after progeny testing (0.8). Therefore, it was assumed that larger bull-teams would be used in dairy herds to reduce the risk of retrospective poor performance by using low reliability sires. In fact, the prediction error variance (PEV) of $10 \mathrm{SC}$ in PT (where $\left.\mathrm{r}^{2}=0.8\right)$ is equivalent to the PEV of $20 \mathrm{SC}$ in GS $\left(\mathrm{r}^{2}=\right.$ $0.6)$, where $\mathrm{PEV}=\left(1-\mathrm{r}^{2}\right) / \mathrm{N}$ (Cameron, 1997) and $\mathrm{N}$ is the number of $\mathrm{SB}$ or $\mathrm{SC}$. In PT, the male candidates for selection are generally the result of planned matings, so SB will generally be less the SC. However, in the GS schemes that include intense selection in male pathways, thousands of males will be genotyped. These are unlikely to be the result of planned matings; therefore, it was assumed $\mathrm{SB}=\mathrm{SC}$ in GS schemes. In schemes that had intense selection in female pathways, there could be more control by the breeding company, which would mean that SB $<$ SC. However, for consistency and to make the results easier to interpret, $20 \mathrm{SB}$ were considered for all GS schemes. The effect of the number of male candidates selected on rate of genetic gain and inbreeding was evaluated for completeness.

\section{Intense Selection in Male Pathways}

$\boldsymbol{G S} \boldsymbol{S} \boldsymbol{Y}$. In this scheme, no progeny testing occurred and all selection of SC was on GEBV. Both SB and $\mathrm{SC}$ were $2 \mathrm{yr}$ of age when their offspring were born, and bulls in the SB and SC pathways were selected using GEBV. For the base scenarios, 1,000 selection candidates were genotyped. Selection in the DB pathway was based on conventional EBV; as with PT and GS-PT, selection across age groups occurred. Again, we assumed no selection in the DC pathway. The effect of sexed semen was investigated for GS-Y (and GS-Y-DC) by changing the sex ratio to $90 \%$ female to $10 \%$ male offspring, which is the sex ratio typically found using flow-cytometric sperm sorting (e.g., DeJarnette et al., 2008).

$\boldsymbol{G} \boldsymbol{S}-\boldsymbol{Y}$ - $\boldsymbol{D} \boldsymbol{C}$. In this scheme, the same assumptions as GS-Y were applied, with the addition of selection in the DC pathway through selection of heifers and cows. One way to generate sufficient replacements is to allow heifers as well as cows to be selection candidates. Therefore, an increase in selection intensity and reduction in generation interval would occur in the DC pathway. The assumptions made were that the replacement rate was $25 \%$ and the calving rate to $\mathrm{AI}$ was $60 \%$ in lactating cows and $70 \%$ in heifers. The replacements generated from lactating cows and heifers would be 8.75 and $22.5 \%$, respectively. The proportion that could be selected in the DC pathway would be 0.8 , calculated as $0.25 /(0.0875+0.225)$. In this scenario, selection occurred in all 4 pathways of selection. Note that the generation interval in the DC pathway is shorter than that in other GS schemes, because heifers and cows are selected as dams of the next generation. 
$\boldsymbol{G} \boldsymbol{S}-\boldsymbol{W} \boldsymbol{W}$. This scheme is called the "worldwide" scheme. It was assumed that GEBV were available on young bulls from 6 countries. Each country had a population of 140,000 cows and had GEBV available on 1,000 (new) males per year. Selection within each country was assumed the same as that in GS-Y. The GEBV mean was equal for each country. Selection of SB and $\mathrm{SC}$ was based on truncation selection on GEBV across all 6 countries, using the same methodology as truncation selection across age classes. In total, 20/6,000 bulls were selected using truncation selection across multiple normal distributions for the SB and SC pathways. The total generation interval was the same as that in GSY. The reliability of GEBV was 0.6 in 1 country (A), which was the country in which benefits to selection were realized. In the other 5 countries, the reliability of GEBV was 0.48 because we assumed that the reliability was $80 \%$ of the reliability of country A. This adjusts the reliability for differences in estimates of SNP effects between countries, on the assumption that reliabilities in the domestic country could be estimated more accurately than that in other countries. This may not be the case if larger reference populations and denser SNP panels are available in other countries.

\section{Nucleus Schemes: Intense Selection in Female Pathways}

GS-CN1. This breeding scheme comprised of a nucleus of 300 heifers. The heifers participated in a JIVET scheme; they became embryo donors at 3 mo of age and were parents at $1 \mathrm{yr}$ of age. According to Raadsma and Tammen (2005), 5 to 10 viable embryos are expected per in vivo harvest. In this scheme, it was assumed that there was an opportunity for only one in vivo harvest. Therefore, on average, 2 viable offspring could be produced per donor (1 male and 1 female). As the nucleus was closed to additional females after its establishment, we assumed no flow of genes from the general population. Therefore, genetic improvement in the DC pathway is equivalent to selection of female replacements in the nucleus. However, in this scenario 300 heifers would be produced per year, which would lead to no selection intensity in the DC pathway. The proportion selected in the male pathways was 20/300 and selection was using GEBV. The total generation interval across 4 pathways of selection was $6 \mathrm{yr}$ (males were $2 \mathrm{yr}$ of age when their offspring were born and females were 1 yr of age).

$\boldsymbol{G S}$ - $\boldsymbol{N}$ 2. This scheme was the same as GS-CN1, except that the age that a heifer would become a parent was increased to $2 \mathrm{yr}$, meaning that there was a greater opportunity to collect more embryos from do- nors. Therefore, it was assumed that 4 viable offspring could be generated per donor. The number of females generated per year was therefore 600 , of which 300 would be selected to become the next generation of the nucleus population; thus, selection occurred in the DC pathway. Selection also occurred in the DB pathway, because half of the females were selected as parents of the next generation. The total generation interval across 4 pathways of selection was $8 \mathrm{yr}$, because the generation interval in each pathway of selection was 2 yr.

\section{Selection Equilibrium}

Selection results in a reduction in genetic variance in selected parents, known as the Bulmer effect (Bulmer, 1971). Using the equations of Bulmer (1971), the genetic variance in selected parents that is passed on to the next generation was calculated as

$$
\begin{aligned}
& k=I\left(I-T_{x}\right) \\
& \sigma_{s}^{2}=\left(1-r^{2} k\right) \sigma_{t}^{2},
\end{aligned}
$$

where $k$ is the variance reduction coefficient, $I$ and $T_{x}$ were defined previously as the selection intensity and truncation point, respectively, $r^{2}$ is the reliability of selection, $\sigma_{t}^{2}$ is the variance in generation $t$, and $\sigma_{s}^{2}$ is the variance in selected parents in generation $t$.

The genetic variance $\left(\sigma_{A}^{2}\right)$ in the next generation was calculated separately for males $\left(\sigma_{A_{-} \text {male }}^{2}\right)$ and females $\left(\sigma_{A_{-} \text {female }}^{2}\right)$ as

$$
\begin{aligned}
& \sigma_{A_{-} \text {male }}^{2}=\frac{1}{4} \sigma_{S B}^{2}+\frac{1}{4} \sigma_{D B}^{2}+\sigma_{M S}^{2}, \\
& \sigma_{A_{-} \text {female }}^{2}=\frac{1}{4} \sigma_{S C}^{2}+\frac{1}{4} \sigma_{D C}^{2}+\sigma_{M S}^{2},
\end{aligned}
$$

where $\sigma_{S B}^{2}$ is the genetic variance in the SB pathway, and so on for SC, DC, and DB; and $\sigma_{M S}^{2}$ is the variance of the Mendelian sampling term assumed to be $\frac{1}{2}$. Selection on a single trait was considered, with annual responses to selection being expressed in genetic standard deviation units.

\section{Rate of Inbreeding}

The rate of inbreeding per generation $(\Delta F /$ gen $)$ and per year $(\Delta F / y r)$ were calculated using the following equations (Gowe et al., 1959): 
Table 3. The annual rate of genetic improvement in $\sigma_{\mathrm{A}}$ units per year $(\Delta \mathrm{G} / \mathrm{yr})$, rate of inbreeding per generation $(\Delta \mathrm{F} /$ generation $)$ and per year $(\Delta \mathrm{F} / \mathrm{yr})$, and percentage annual rate of inbreeding compared with $\mathrm{PT}(\Delta \mathrm{F} /$ year relative to $\mathrm{PT})$

\begin{tabular}{lccccc}
\hline Scheme $^{1}$ & $\begin{array}{c}\Delta \mathrm{G} / \mathrm{yr} \\
\left(\sigma_{\mathrm{A}} / \mathrm{yr}\right)\end{array}$ & $\begin{array}{c}\Delta \mathrm{G} / \mathrm{yr} \text { relative } \\
\text { to PT }(\%)\end{array}$ & $\Delta \mathrm{F} /$ generation & \multicolumn{2}{c}{$\Delta \mathrm{F} / \mathrm{yr}$ relative } \\
to PT $(\%)$
\end{tabular}

${ }^{1}$ See Table 1 for a description of each scheme.

$$
\begin{aligned}
& \Delta F / \text { gen }=\frac{1}{32 L}\left[\frac{1}{N_{S B}}+\frac{3}{N_{S C}}+\frac{1}{N_{D B}}+\frac{3}{N_{D C}}\right], \\
& \Delta F / y r=\frac{\Delta F / g e n}{L},
\end{aligned}
$$

where $L$ is the generation interval (i.e., average age of parents when their offspring are born) and $N_{S B}$ is the number of new SB per generation, and so on for SC, DC, and DB.

\section{RESULTS AND DISCUSSION}

The results presented here are based on one round of selection (with the exception of Figure 4). The response to selection $(\Delta \mathrm{G})$ for PT was $0.25 \sigma_{\mathrm{A}} / \mathrm{yr}$ (Table 3 ). This is similar to the deterministic prediction of response in PT reported by Schaeffer (2006) and the realized rate reported by Van Doormaal and Kistemaker (2003). In GS-PT, 50 out of 1,000 genotyped bulls were selected for inclusion in the progeny test team based on GEBV. In this scheme, bulls were only selected as AI sires when they had progeny test daughters. The GS-PT scheme led to a modest increase in response to selection of $16 \%$ more than progeny testing. The same rate of genetic gain as PT can be achieved through screening 120 bulls using GEBV and selecting the best 50 for progeny testing. Savings can obviously be made by reducing the number of sires that are put forward to progeny testing (Schaeffer, 2006), and our results demonstrate that even conservative adaptations to breeding schemes using GEBV can be beneficial.

\section{Young Bull Schemes}

Reducing the generation interval had the greatest effect on response to selection (Table 3). Scheme GS$\mathrm{Y}$, where bulls were selected for widespread use in the industry at $1 \mathrm{yr}$ of age based on their GEBV, led to a large increase in $\Delta \mathrm{G}\left(0.40 \sigma_{\mathrm{A}} / \mathrm{yr}\right)$. The response to se- lection was $59 \%$ greater than the response to selection of PT. The sum of generation intervals in each pathway of selection for GS-Y was $12.3 \mathrm{yr}$ compared with $21.3 \mathrm{yr}$ for PT and GS-PT (Table 2).

Although little selection generally occurs in the DC pathway (Spelman and Garrick, 1997), some gains can be made from increasing the selection intensity and reducing the generation interval in this pathway. Scheme GS-Y-DC was an extension of the GS-Y scheme that included selection in the DC pathway. This was feasible because heifers as well as cows were available as selection candidates. It was assumed that $80 \%$ of cows and heifers were selected as dams of the next generation and that phenotypic records were available on all animals. The improvement in response to selection of GS-Y-DC compared with GS-Y was $+17 \%$. If only cows (rather than heifers and cows) were selected as DC, then the corresponding increase in response would be $+3 \%$.

One way to increase the selection response further is to use GEBV for female selection pathways, which would increase the accuracy of young females in particular. The rate of response assuming GEBV were used in all 4 pathways of selection would be $0.54 \sigma_{\mathrm{A}} /$ yr. This is a $+14 \%$ improvement over GS-Y-DC and a $+33 \%$ improvement on GS-Y. If GEBV were available in 3 pathways of selection (SB, SC, and $\mathrm{DB}$ ) then the response to selection would be $0.53 \sigma_{\mathrm{A}} / \mathrm{yr}$. Therefore, having GEBV in the DB pathway is much more beneficial than having GEBV in the DC pathway, because the difference between these schemes was only 0.01 $\sigma_{\mathrm{A}} / \mathrm{yr}$. Furthermore, the cost of genotyping at present means that it would not be economically worthwhile for a farmer to genotype a herd of lactating dairy cattle, because the cost would likely far exceed the benefit (Schaeffer, 2006; König and Swalve, 2009).

\section{Breeding Schemes that Include Several Countries}

Scheme GS-Y was extended to include 6 countries (GS-WW), and it was assumed GEBV were available for all 6 countries. Genotype by environment interactions 
of 5 of the countries were accounted for by multiplying the reliability assumed of GEBV by 0.8 ; that is, assuming GMACE is available (VanRaden and Sullivan, 2010). The response to selection in GS-WW was 0.41 $\sigma_{\mathrm{A}} / \mathrm{yr}$, which is $2.5 \%$ higher than that in GS-Y.

If GMACE is relied upon to generate GEBV, then marginally higher gains can be made than are achieved within country (i.e., comparing GS-Y to GS-WW). However, if we assumed that the reliabilities of all 6 participating countries were equal (0.6), which is feasible if genotypes were freely exchanged, then the response to selection would be $0.48 \sigma_{\mathrm{A}} / \mathrm{yr}$. This is close to double the rate of genetic gain achieved in $\mathrm{PT}$ and $17 \%$ greater than GS-Y.

In GS-WW, most of the bulls $(93.5 \%)$ were selected from the country with the highest reliability for GEBV; $1.3 \%$ of bulls would be selected from each of the 5 remaining countries. If the GEBV reliability of all countries were assumed to be the same, equal proportions of bulls would be selected from each country (16.7\%). If one country is genetically ahead of the others by 1 generation of selection and the reliabilities of GEBV in all countries are equal, then $46.2 \%$ of bulls would be selected from that country and $10.7 \%$ would be selected from each of the others.

Scheme GS-WW requires little organization other than provision of GEBV of foreign bulls on a domestic scale. If exchange of genotypes occurs, then achieving the same reliabilities for participating countries is possible provided that unbiased GEBV can be estimated for each country. This approach would require a sufficiently large reference population in each country to derive an accurate prediction equation, and, of course, use of a common SNP panel to predict GEBV. This type of scheme will be particularly beneficial for countries with small populations of dairy cows, especially where there are other countries with populations of dairy cattle that are at least as good as the importing country. Alternatively, it may be of interest in countries that lack a national breeding scheme. It could also be used by minor breeds where selection decisions within country are constrained by population size.

\section{Nucleus Breeding Schemes}

Very high rates of genetic gain can be achieved by implementing nucleus breeding schemes. The response to selection of GS-CN1 was $0.50 \sigma_{\mathrm{A}} / \mathrm{yr}$ and that of GSCN was $20.59 \sigma_{\mathrm{A}} / \mathrm{yr}$ (Table 3). Schemes GS-CN1 and GS-CN2 demonstrated that very high rates of genetic gain could be achieved if reproductive technologies are used aggressively to shorten the generation interval and increase the number of calves born per dam. The generation interval in female pathways of selection of
GS-CN1 was 1 yr, and for GS-CN2 was 2 yr (Table 2). However, it was assumed that JIVET could not generate as many offspring in GS-CN1 as in GS-CN2 because of biological limitations in 3-mo-old heifers and insufficient time for more than one collection of embryos. Because GS-CN1 and GS-CN2 are heifer-only schemes, they could be operated by complete replacement of the herd on an annual basis, which may become costly if heifers are purchased. An alternative would be to have a dispersed nucleus where heifers are enrolled in JIVET but remain the property of the farmer.

An obvious opportunity with initiating a nucleus herd is that it could become a cooperative central-testing unit where cows are enrolled to obtain unbiased phenotypic data. These units could provide elite females as potential bull dams, and well-recorded phenotypes could be used to re-estimate SNP effects routinely. Regular addition of phenotypic records on milk production and health and fertility records for daughters of genotyped bulls will be important, because the relationships between genotypes and phenotypes will need to be re-estimated regularly to avoid a decline in reliability (e.g., Habier et al., 2007). Provided that farmers are willing to cooperate with breeding companies in collecting phenotypic data on selected individuals and their contemporaries, it may be possible to set up a dispersed nucleus where cows remain the property of individual farmers. However, preferential treatment of selected cows may become an issue in dispersed nuclei.

Another option is to replace test stations of bull dams with a large number of phenotypic records in cooperator progeny-test herds. König and Swalve (2009) argued that adding phenotypic records would increase the reliability of EBV for low heritability traits (based on phenotype plus genotype information) to levels the market would accept while saving costs. However, the limitation with this approach is that there would be no improvement in the rate of genetic gain.

\section{Inbreeding}

As more sires would be generated from fewer cows using GS-CN1, there is a risk that the rate of inbreeding may become prohibitive. Comparing the rate of genetic gain of various breeding scheme designs was the focus of this study; however, rates of inbreeding were also compared (Table 3 and Figure $3 \mathrm{~b}$ ). The calculation of inbreeding ignored the effects of selection. Detailed descriptions of how to implement these methods are given by Bijma and Woolliams (2000) and Rutten et al. (2002) among others. However, Wray and Thompson (1990) stated that predictions that do not consider selection (such as the method used here) are more easily attainable and are unlikely to affect 
the ranking of alternative breeding schemes with respect to $\Delta \mathrm{F}$. Furthermore, quantifying inbreeding for breeding schemes accurately is complicated by ad hoc inbreeding control measures. Often breeding companies have diverse breeding goals, such as high fertility or perceived desirable conformation that might increase genetic diversity and reduce inbreeding. Therefore, the inbreeding results here should be considered as a way of comparing breeding schemes rather than an expectation of $\Delta \mathrm{F}$.

Compared with the rate of inbreeding expected in PT, GS-CN1 and GS-CN2 would increase the rate of inbreeding by 165 and $93 \%$ respectively (Table 3 ). However, GS-Y, GS-Y-DC, and GS-WW actually had lower rates of inbreeding per year and per generation than did PT. Inbreeding is primarily a function of number selected rather than population size (Falconer, 1989; Weigel, 2001). The reason why the genomic selection schemes (except for nucleus breeding schemes) had lower rates of inbreeding than PT was mainly because of the number of males selected as parents of the next generation, especially in the SB pathway. We assumed that there were $2 \mathrm{SB}$ in progeny-testing selected out of 100 candidate sires. If the number of SB were equal to SC (10), then the annual rate of inbreeding for PT would be lower than all genomic selection breeding schemes. In Figure $3 \mathrm{~b}$ it can be seen that the rate of inbreeding decreases as the number of sires (SB and SC) increases. Scheme GS-CN1 is particularly sensitive to the number of $\mathrm{SB}$ and $\mathrm{SC}$ in both the response to selection and the rate of inbreeding (Figure $3 \mathrm{a}$ and $3 \mathrm{~b}$ ).

Future work could include optimizing the rate of genetic gain in GS schemes while constraining inbreeding to an acceptable level, similar to Wray and Goddard (1994), who considered conventional schemes. Such strategies could include minimizing the genetic relationships between individuals or maximizing heterozygosity in SNP that are not under direct selection; this would be especially beneficial if these SNP are assumed to be in linkage disequilibrium with (not-measured) dairy fitness traits.

\section{Effect of Reliability}

The response to selection was greatest in GS-CN2 except for very low GEBV reliabilities $(<0.2)$ where the $\Delta \mathrm{G} / \mathrm{yr}$ was highest in GS-Y-DC (Figure 1). When the reliabilities of GEBV were $>0.5$, both nucleus breeding schemes (GS-CN1 and GS-CN2) had higher $\Delta \mathrm{G} /$ yr than all of the other schemes. Schemes GS-Y and GS-WW had the same rate of increase in response with increasing reliability. A larger $\Delta \mathrm{G} / \mathrm{yr}$ than GS-PT was achieved when reliabilities were $>0.1$, except for GSYCC, where selection in female pathways resulted in a response greater than GS-PT for all GEBV reliabilities investigated $(\geq 0.1)$.

\section{Number of Animals Genotyped}

In the male schemes, the benefit of genotyping extra individuals is constrained by the total population size. For example, in Figure 2, $\Delta \mathrm{G} / \mathrm{yr}$ is shown against the number of animals genotyped when the population was 140,000 breeding females for GS-Y and GS-Y-DC. The response appears to reach a plateau as more bulls were genotyped. The difference between GS-Y and GS-YDC remains constant as the number of bulls genotyped increases.

To investigate the effect of the size of population of breeding females, GS-Y was increased by 10 -fold to 1.4 million (GS-Y-10 $\times$ ). The difference in $\Delta \mathrm{G} /$ year in a population of 140,000 versus 1.4 million (GS-Y and GS-Y-10× respectively) is greater when 10,000 bulls are genotyped than 1,000. This demonstrates the effect of using all females in the population rather than the pedigree-registered cows. Currently, most breeding companies select bull dams from available dairy cows with known recorded ancestry. Genomic selection can be used to increase the population of selection candidates by extending the population to include commercial cows. The main reason why the response to selection of GS-Y-DC exceeded that of GS-Y-10× was that the generation interval was smaller in GS-Y-DC because heifers as well as cows were selected in the DC pathway.

\section{Selection to an Asymptotic State}

One of the advantages of stochastic simulation over deterministic selection is that many rounds of selection can be simulated, which should result in a more accurate estimate of variance and response. However, the Bulmer effect of variance reduction through selection can be modeled deterministically over successive generations of selection until an asymptotic state is reached (Bulmer, 1971).

To investigate the effect on $\Delta \mathrm{G}$ of multiple generations of the Bulmer effect, responses to selection over 10 generations of selection were calculated. The annual rates of genetic gain following 1 round of selection are presented elsewhere in this paper (e.g., Table 3). In most cases, the ranking of the schemes was preserved when comparing annual rates of genetic gain after 1 round of selection versus genetic gain after the asymptote was reached (Figure 4). The asymptote was reached after about 5 generations of selection, at which point the reduction in selection response was between $9.9 \%$ (PT) and 21\% (GS-CN1) compared with single-round 


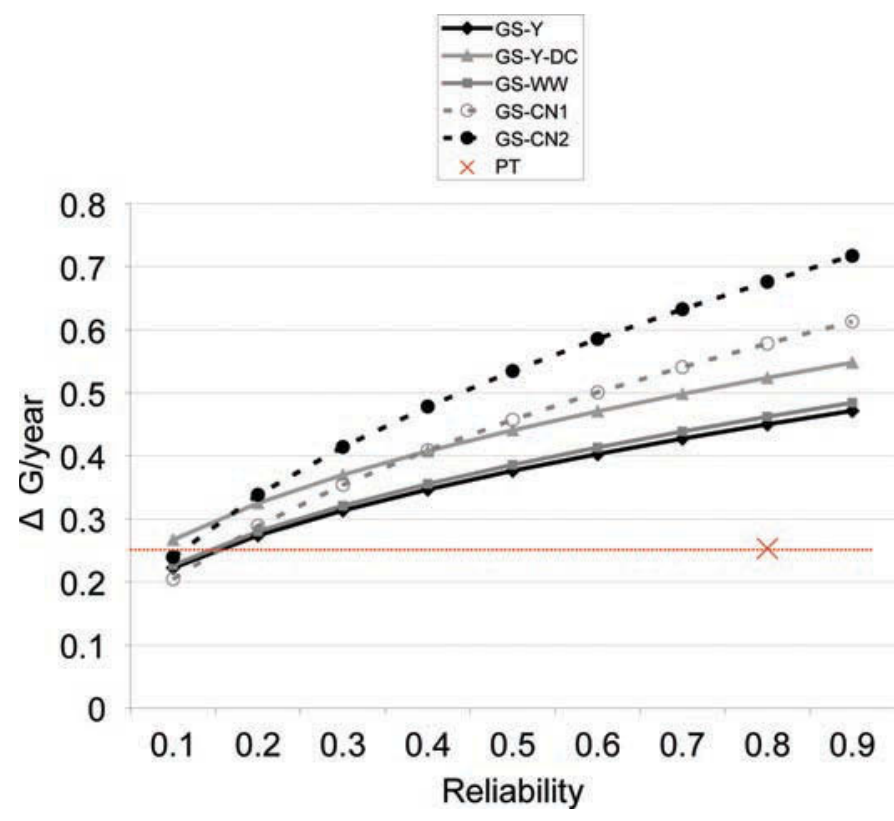

Figure 1. Response to selection achieved in progeny testing (PT) and the effect on response to selection per year $\left(\Delta \mathrm{G} / \mathrm{yr}\right.$ in $\left.\sigma_{\mathrm{A}} / \mathrm{yr}\right)$ from varying the reliability of genomic EBV in genomic selection schemes: GS-Y, GS-WW, GS-Y-DC, GS-CN1, and GS-CN2. The reliability and response currently achieved under PT is denoted by $\times$. Refer to Table 1 for abbreviations and brief descriptions of breeding schemes. Color version available in the online PDF.

selection. After reaching the asymptote, the difference in $\Delta \mathrm{G}$ between some schemes decreased. For example, after 10 generations, the difference between GS-Y-DC and GS-Y decreased from 0.068 to $0.039 \sigma_{\mathrm{A}} / \mathrm{yr}$ (Figure 4). This was because more variance reduction occurred in the GS-Y-DC scheme due to the selection intensity in the DC pathway. It should be remembered that the generation intervals differed between schemes: $L$ was 2 yr for GS-CN1 and 5.3 yr for PT.

\section{Sexed Semen}

The use of sexed semen could have a detrimental effect on breeding schemes that have selection pressure in male pathways of selection because, in commercial situations, farmers are likely to use sexed semen to increase the ratio of females to males. The implication of this is that fewer male candidates would exist for selection. If the sex ratio became $90 \%$ female to $10 \%$ male and there was no selection in the DC pathway, then the response to selection in GS-Y would reduce by $10 \%$ (which is a $\Delta \mathrm{G}$ of 0.37 ). However, it is obvious that using sexed semen will result in selection in the female pathways of selection, and thus the impact of sexed semen in the GS-Y-DC breeding scheme should give a better idea of the impact of sexed semen on the rate of genetic gain.
In GS-Y-DC, if sexed semen were used to generate female replacements, assuming a sex ratio of $90 \%$ females and a replacement rate of $25 \%, \Delta \mathrm{G}$ would be $0.53 \sigma_{\mathrm{A}} / \mathrm{yr}$. This is an improvement of $13 \%$ over the $\Delta \mathrm{G}$ achieved in GS-Y-DC without sexed semen, suggesting that sexed semen in conjunction with genomic selection could realize even higher rates of genetic gain. In conventional breeding scheme designs, the advantage of sexed semen may be approximately $11 \%$ compared with a scheme where nonsexed semen is used (Abdel-Azim and Schnell, 2007).

Similar improvements can be achieved by using sexed semen in nucleus breeding schemes. By altering the sex ratio to be $33 \%$ male in GS-CN1, $\Delta \mathrm{G}$ can be increased from 0.50 to $0.56 \sigma_{\mathrm{A}} / \mathrm{yr}$ (an improvement of $12 \%$ ). Note that this sex ratio is very close to the maximum rate of genetic gain achievable in GS-CN1 solely through altering the sex ratio.

In the scenarios described, we assumed that the fertility of sexed semen was equal to that of conventional semen. Unfortunately, only a limited amount of data exists on conception rates achieved using sexed semen. Currently, the method used for most commercial sexing of semen is flow cytometry (Johnson et al., 1989), which may be detrimental to sperm viability and quality. Lower conception rates have been found in heifers, which can be partly compensated for by increasing the sperm dosage (DeJarnette et al., 2008). Future advances in sexed semen technology may result in similar conception rates to those achieved with conventional semen.

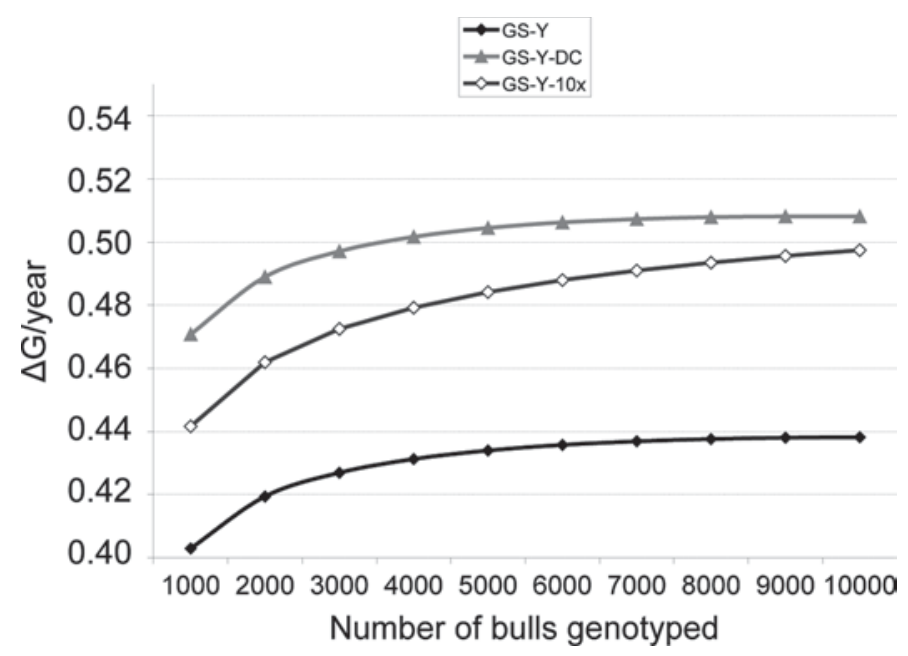

Figure 2. Response to selection per year $\left(\Delta \mathrm{G} / \mathrm{yr}\right.$ in $\left.\sigma_{\mathrm{A}} / \mathrm{yr}\right)$ achieved by varying the number of candidates genotyped from 1,000 to 10,000 when the total population of breeding females is 140,000 (GS-Y and GS-Y-DC) and for GS-Y when the female breeding population is increased 10-fold to 1.4 million (GS-Y-10×). The number of bulls selected is 20 in all scenarios. Refer to Table 1 for abbreviations and brief descriptions of breeding schemes. 


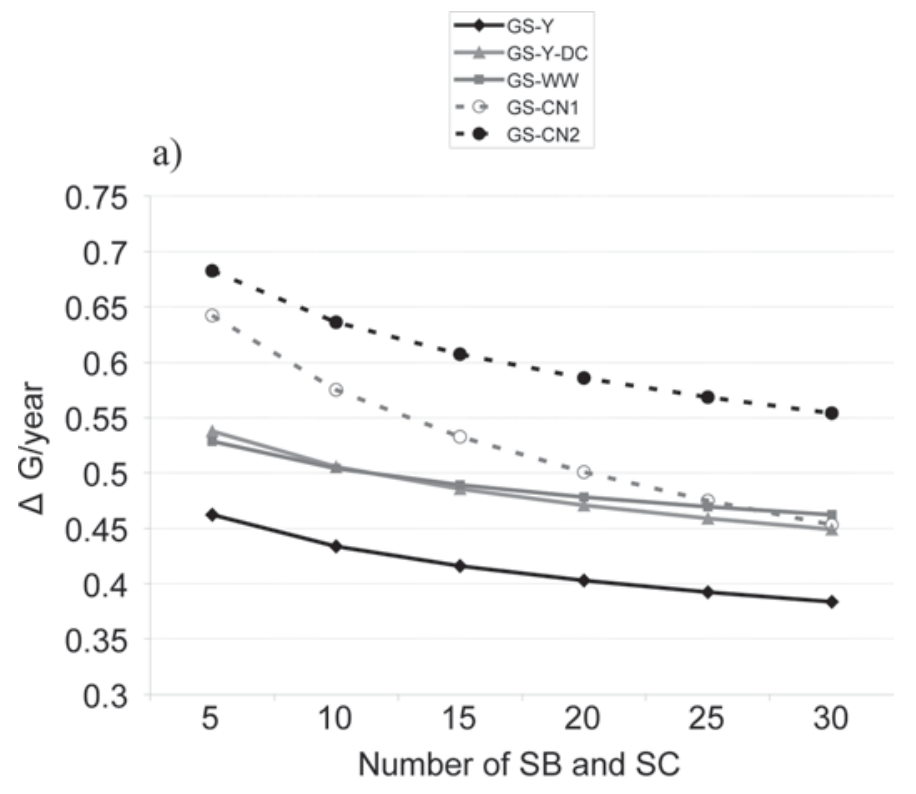

b)

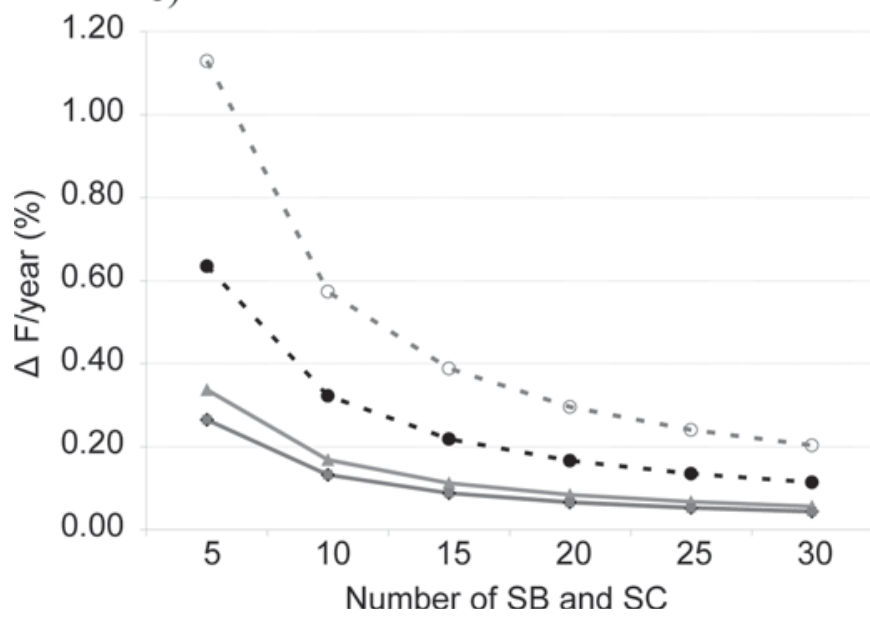

Figure 3. a) Response to selection per year $\left(\Delta \mathrm{G} / \mathrm{yr}\right.$ in $\left.\sigma_{\mathrm{A}} / \mathrm{yr}\right)$ and b) rate of inbreeding per year $(\Delta \mathrm{F} / \mathrm{yr}$ in $\%)$ achieved by varying the number of candidates selected as sires of bulls (SB) and sires of cows (SC) from 5 to 30 in genomic selection schemes: GS-Y, GS-WW, GSY-DC, GS-CN1, and GS-CN2. Refer to Table 1 for abbreviations and brief descriptions of breeding schemes.

\section{Maintenance of Training Populations}

Accuracy of GEBV is generally validated by correlating GEBV calculated using prediction equations estimated from training data with EBV from young sires as soon as progeny data are available (e.g., Hayes et al., 2009). However, under the scenarios suggested here, routine progeny testing of hundreds of sires under current PT schemes would be replaced with testing 20 sires. This presents challenges in defining future validation populations. In addition to ensuring that all major male ancestors with DNA samples available are genotyped, the training population could be increased by large-scale genotyping of females.

\section{CONCLUSIONS}

All breeding schemes using genomic selection considered here achieved greater rates of response than conventional progeny-testing schemes, even when a mixture of genomic selection and progeny testing was used. Nucleus schemes and "worldwide" schemes, where GEBV were available from foreign countries, achieved the greatest responses to selection. Even greater responses were achieved if reproductive technologies in juvenile females were used. This demonstrates that the greatest gains in genomic selection applied to dairy cows are achieved by reducing the generation interval. However, in nucleus schemes, such gains have to be offset against potentially negative effects of greatly increased levels of inbreeding. In other genomic selection schemes, such as GS-Y, levels of inbreeding per year are actually likely to decrease by up to $50 \%$. It is likely that breeding companies will move rapidly to breeding schemes using genomic selection because the cost efficiencies for the companies and the industry-wide benefits are considerably higher than those currently achieved under progeny-testing schemes.

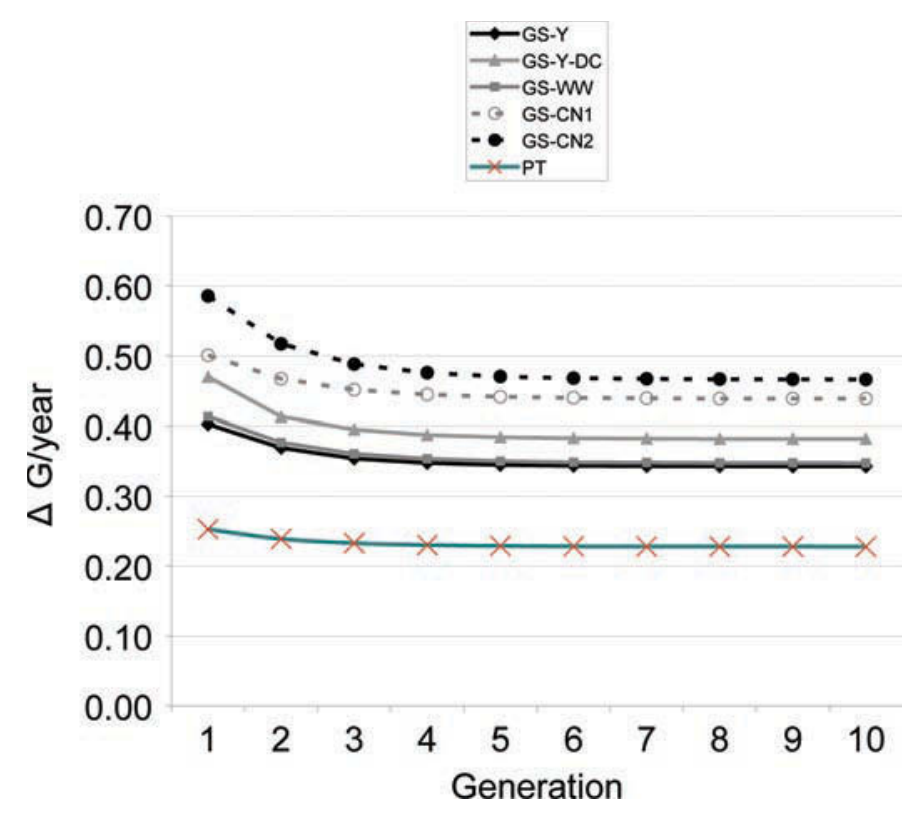

Figure 4. Response to selection $\left(\Delta \mathrm{G} / \mathrm{yr}\right.$ in $\left.\sigma_{\mathrm{A}} / \mathrm{yr}\right)$ over 10 generations of selection in progeny testing $(\mathrm{PT})$ and genomic selection schemes: GS-Y, GS-WW, GS-Y-DC, GS-CN1, and GS-CN2. Abbreviations can be found in Table 1. Color version available in the online PDF. 


\section{ACKNOWLEDGMENTS}

Funding was provided through the Dairy Futures Cooperative Research Centre (Melbourne, Australia). We thank the anonymous reviewers of this paper for useful suggestions.

\section{REFERENCES}

Abdel-Azim, G., and S. Schnell. 2007. Genetic impacts of using female sorted semen in commercial and nucleus herds. J. Dairy Sci. 90:1554-1563.

Bijma, P., and J. A. Woolliams. 2000. Prediction of rates of inbreeding in populations selected on best linear unbiased prediction of breeding value. Genetics 156:361-373.

Bulmer, M. G. 1971. The effect of selection on variability. Am. Nat. 105:201-211.

Cameron, N. D. 1997. Selection indices and prediction of genetic merit in animal breeding. CAB International, Wallingford, UK.

Cunningham, E. P. 1975. Multistage selection. Theor. Appl. Genet. 46:55-61.

Dairy Research and Development Corporation. 2000. The in-calf project-A progress report. DRDC, Melbourne, Australia.

DeJarnette, J. M., R. L. Nebel, C. E. Marshall, J. F. Moreno, C. R McCleary, and R. W. Lenz. 2008. Effect of sex-sorted sperm dosage on conception rates in Holstein heifers and lactating cows. J. Dairy Sci. 91:1778-1785.

Ducrocq, V., and R. L. Quaas. 1988. Prediction of genetic response on truncation selection across generations. J. Dairy Sci. 71:25432553.

Falconer, D. S. 1989. Introduction to Quantitative Genetics. 3rd ed. Longman Scientific and Technical, New York, NY.

Goddard, M. E. 2009. View to the future: Could genomic evaluation become the standard. Interbull Bull. 39:83-88.

Gowe, R. S., A. Robertson, and B. D. H. Latter. 1959. Environment and poultry breeding problems. 5. The design of poultry control strains. Poult. Sci. 38:462-471.

Habier, D., R. L. Fernando, and J. C. Dekkers. 2007. The impact of genetic relationship information on genome-assisted breeding values. Genetics 177:2389-2397.

Haile-Mariam, M., J. M. Morton, and M. E. Goddard. 2003. Estimates of genetic parameters for fertility traits of Australian HolsteinFriesian cattle. Anim. Sci. 76:35-42.

Harris, B. L., D. L. Johnson, and R. J. Spelman. 2008. Genomic selection in New Zealand and the implications for national genetic evaluation. Proc. 36th ICAR Biennial Session, Niagara Falls, NY. ICAR, Rome, Italy.

Hayes, B. J., P. J. Bowman, A. J. Chamberlain, and M. E. Goddard 2009. Genomic selection in dairy cattle: Progress and challenges. J. Dairy Sci. 92:433-443.
Johnson, L. A., J. P. Flook, and H. W. Hawk. 1989. Sex pre-selection in rabbits: Live births from X and Y sperm separated by DNA and cell sorting. Biol. Reprod. 41:199-203.

Jopson, N. B., P. R. Am, and J. C. McEwan. 2004. Comparison of twostage selection breeding programmes for terminal sire sheep. Proc. N.Z. Soc. Anim. Prod. 64:213-216.

König, S., H. Simianer, and A. Willam. 2009. Economic evaluation of genomic breeding programs. J. Dairy Sci. 92:382-391.

König, S., and H. H. Swalve. 2009. Application of selection index calculations to determine selection strategies in genomic breeding programs. J. Dairy Sci. 92:5292-5303.

Meuwissen, T. H. E., B. J. Hayes, and M. E. Goddard. 2001. Prediction of total genetic value using genome-wide dense marker maps. Genetics 157:1819-1829.

Miglior, F., B. L. Muir, and B. J. Van Doormaal. 2005. Selection indices in Holstein cattle of various countries. J. Dairy Sci. 88:12551263

Moser, G., B. Tier, R. E. Crump, M. S. Khatkar, and H. W. Raadsma 2009. A comparison of five methods to predict genomic breeding values of dairy bulls from genome-wide SNP markers. Genet. Sel. Evol. 41:56

Raadsma, H. W., and I. Tammen. 2005. Biotechnologies and their potential impact on animal breeding and production: A review. Aust. J. Exp. Agric. 45:1021-1032.

Rendel, J. M., and A. Robertson. 1950. The use of progeny testing with artificial insemination in dairy cattle. J. Genet. 50:21-31.

Rutten, M. J. M., P. Bijma, J. A. Woolliams, and J. A. M. van Arendonk. 2002. SelAction: Software to predict selection response and rate of inbreeding in livestock breeding programs. J. Hered. 93:456-458

Schaeffer, L. R. 2006. Strategy for applying genome-wide selection in dairy cattle. J. Anim. Breed. Genet. 123:218-223.

Spelman, R. J., and D. J. Garrick. 1997. Utilisation of marker assisted selection in a commercial dairy cow population. Livest. Prod. Sci 47:139-147.

Van Doormaal, B. J., and G. J. Kistemaker. 2003. Dairy genetic improvement through artificial insemination, performance recording and genetic evaluation. Can. J. Anim. Sci. 83:385-392.

VanRaden, P. M., and P. G. Sullivan. 2010. International genomic evaluation methods for dairy cattle. Genet. Sel. Evol. 42:7.

VanRaden, P. M., C. P. Van Tassell, G. R. Wiggans, T. S. Sonstegard, R. D. Schnabel, J. F. Taylor, and F. S. Schenkel. 2009. Invited review: Reliability of genomic predictions for North American Holstein bulls. J. Dairy Sci. 92:16-24.

Weigel, K. A. 2001. Controlling inbreeding in modern breeding programs. J. Dairy Sci. 84(E. Suppl.):E177-E184.

Wray, N. R., and M. E. Goddard. 1994. Increasing long-term response to selection. Genet. Sel. Evol. 26:431-451.

Wray, N. R., and R. Thompson. 1990. Prediction of rates of inbreeding in selected populations. Genet. Res. 55:41-54. 